\title{
AMCP's Annual Meeting: Building a Bridge to a Successful Future
}

\section{Two keynote speakers shared with Annual Meeting attendees insightful views}

on managed care, its future, and pharmacy's place in it.
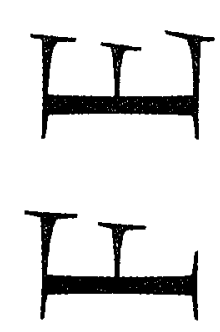

\section{KAREN SOUTHWICK}

KAREN SOUTHWICK is a San Francisco-based contributing writer for Pharmacare Economics, a supplement to Managed Healthcare.

Copyright @ 1996 Academy of Managed Care Pharmacy, Inc. All rights reserved.
$\mathrm{L}$ aunching JMCP's coverage of the Academy of Managed Care Pharmacy's Eighth Annual Meeting is this summary of the two keynote speakers. The first offered a virtual reality trip into the future, while the second based his analysis on hard-nosed, rough-andready experience. Here is what they said in San Francisco.

\section{MANAGED CARE IN METRO MARKETS}

The Clinton health care reform effort may have resembled a Gilbert $\&$ Sullivan operetta, but it did leave one important legacy: frightening health care providers and suppliers into huge economic concessions that continue to lower the cost of care.

That was the view of Jeff $C$. Goldsmith, Ph.D., a health futurist who gave the first keynote address at the Eighth

Continued on page 364 
Annual Meeting of the Academy of Managed Care Pharmacy in San Francisco. Goldsmith, president of Health Futures Inc, in Bannokburn, Ill., said the decline in premium costs has resulted "in a lot of organizational scrambling around."

Health care providers almost unanimously agree, he explained, that the future shape of the metropolitan health care environment will include three to six vertically integrated, sole-source, full-spectrum providers from which payers and consumers will choose. Such vertically integrated providers will be fully capitated and employ physicians and other needed providers.

This view persists despite the fact that "consumers are telling us they don't want closed panels. They want broad inclusive networks and they want to make the decisions about who will provide their care. They want to continue relationships with hospitals and doctors who have served them well, and they want database information with which to make those decisions," Goldsmith said.

\section{VERTICAL VS. VIRTUAL INTEGRATION}

Health care is too complicated for the vertical integration model, borrowed from more mature industries, such as automobiles, to function well. "A $\$ 3$ billion enterprise is no better or cheaper than a smaller entity of loosely linked networks," he said. In fact, "diseconomies of scale" occur when giant entities attempt to deliver cost-effective care at the patient level.

As evidence, he points out that staff-model HMOs such as Kaiser Permanente and Group Health Cooperative of Puget Sound, which own hospitals and employ physicians, are now disassembling that model to some extent. They are contracting with hospitals and physicians outside their own plan because they can get quality care more cost effectively.

Goldsmith said what must occur in health care is not vertical integration, but "virtual integration." He defines the latter this way: "Strategic collaboration based on developing and marketing products through contractual arrangements rather than common ownership." By sharing economic risk and sharing values across the partnership, these virtually integrated organizations will become the dominant framework for delivery of care, Goldsmith said.

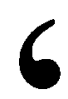

What must occur in health care is not vertical integration, but "virtual integration.

\section{-Jeff C. Goldsmith

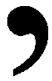

The old model under which managed care first emerged will no longer work, he warned. For one thing, in 1980 , as managed care was just entering its boom cycle, it enrolled only the healthiest people. Now, the sickest members of society at highest risk are participating in managed care, such as Medicaid and Medicare patients.

Second, managed care formerly operated under the high-priced umbrella of "totally unmanaged" indemnity insurance. Such is no longer the case. "Indemnity insurance is a dying business," Goldsmith maintained, and managed care "has won the battle. It is no longer the counterculture force." That means instead of merely being cheaper than somebody else, it must demonstrate value.

Finally, the savings that managed care previously achieved by lowering hospital stays and passing on the reduced costs in the form of lower premiums "is less and less viable as a strategy." Hospitalization rates have dropped among all providers, and the gap between $\mathrm{HMO}$ and other providers' premiums has narrowed. In some cases, PPO prices are even lower than HMOs' because of steep discounts offered by "panicked physicians and hospitals who don't want to be left out," Goldsmith said.

\section{IMPLICATIONS FOR THE FUTURE}

What does all this mean for managed care plans? To survive the coming shakeout, they must move away from managing price toward cultivating consumer satisfaction and away from component management toward risk management. "Instead of waiting until someone gets sick, you try to manage that sickness in a proactive way," he said. One step is to set up means of identifying high-risk populations-and then to develop protocols for lessening that risk.

This prospective rather than eventbased approach will focus on continuous low-intensity interaction with patients and their families, Goldsmith explained. And the health care provider team will include, but not be dominated by, a physician as well as other caregivers such as pharmacists. This team will develop protocols and clinical pathways and create a "seamless continuity of care." No longer will consumers see their interaction with the health care system "as one huge succession of dropped batons," he quipped.

Pharmacists will be important in this new system. They have opportunities to encourage behavioral change and monitor appropriate use of medications in a way that will avoid hospital and nursing-home stays, Goldsmith said. "I see use of pharmaceuticals increasing as we move into this risk-driven, rather than event-driven, world," he said.

Goldsmith, a noted proponent of medical innovation, said biotechnology and genetic diagnosis will enable earlier intervention before diseases become symptomatic: "Pharmacy has a crucial role to play, not only in educating patients about these developments, but in informing the decision-making framework and applying pharmaceutical innovation to improve people's lives."

\section{KNUDSEN: CHARACTERISTICS OF A SUCCESSFUL SYSTEM}

The second keynote speaker, Kermit B. Knudsen, M.D., did not wholly agree with Goldsmith's contentions 
about the problems of vertical integration. Knudsen is a long-time executive with the Scott and White system in Temple, Texas, which employs 470 physicians and operates a hospital, 16 clinics, and an HMO with 120,000 members.

A successful integrated system, he said, aligns the financial incentives of physicians, hospitals, and health plans; provides good communications and information systems; and has access to capital and the ability to shift financial resources. It can also enter into contracts with other health plans or large employers. Formed in 1896 by two surgeons with the Santa Fe Railroad, Scott and White has grown into a dominant health care delivery force in central Texas-where it covers about 16 counties. Besides its own system, it contracts with outside hospitals and independent physician groups.

\section{DEVELOPMENT OF MANAGED CARE OPTION}

Not that Scott and White was always enlightened about managed care. In the early 1970s, it rejected the option of beginning an HMO, terming it "a socialistic idea that would never work," said Knudsen, who has served as president of the Scott and White Clinic and chief of staff of the hospital. Fortunately, he said, wiser thinking prevailed, and in 1982 Scott and White launched a group-model HMO.

Integrated systems can provide the comprehensive care that payers want, Knudsen asserted, by:

$\Delta$ Taking overall responsibility for the coordination and delivery of consistent, appropriate, quality care;

A Assuming overall control of all costs;

$\Delta$ Accepting financial risk; and

$\boldsymbol{\Delta}$ Documenting quality by measuring patient outcomes.

In addition, a well-run integrated system has the capital available to fund all the shifts going on in health care, including inpatient to outpatient, specialist to primary care, acute care to diag- nosis and prevention, and clinical technologies to information systems. "In the next decade we will spend much more on information technology than on MRI

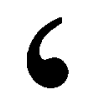

\section{A well-run integrated system has the capital available to fund all the shifts going on in health care}

\section{-Kermit B. Knudsen

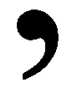

or other fancy gadgets for clinical use," he said.

Scott and White considered joining a large for-profit health care organization several years ago, but ultimately rejected that notion, Knudsen said. The advantages would have been national name recognition, access to capital, special expertise, and additional funding for research and education. However, the disadvantages-such as loss of local control, culture clash, and requirements for maximizing shareholder value-offset those.

\section{PHARMACY: AN INTEGRAL TEAM MEMBER}

What is key to Scott and White is the concept of teamwork, Knudsen said. Although physicians are respected for their input and leadership, they are not the "captains of the ship." Rather, they serve on a multidisciplinary team that includes nurses, pharmacists, and other caregivers. "There's no individual power positions and an absence of departmental fiefdoms," he maintained.

How does the pharmacy department relate to this concept? Under pharmacy head Bill Woodward, Scott and White's pharmacy department has grown from a small hospital-based unit employing six pharmacists back in 1978 to a fully integrated system span- ning inpatient and outpatient care with 150 FTEs. Last year, its budget was $\$ 50$ million.

Knudsen said the pharmacy department at Scott and White also runs under the team concept. Patient-care teams include pharmacists, nurses, and physicians. Six clinical pharmacists meet daily with medical staff and nurses. Pharmacists serve with physicians on a drug therapy task force that achieved $\$ 1.5$ million in savings last year.

Scott and White's portfolio of pharmacy services include outpatient prescription dispensing; clinics in anticoagulation, nephrology/dialysis, and psychiatry; programs in compliance and preventive health; and home intravenous therapy. This year, the pharmacy department is considering starting a women's clinic and a lipid-management clinic.

Advantages of an integrated pharmacy department include:

$\Delta$ Financial stability;

$\Delta$ Flexibility to shift and share staff positions across the hospital, managed care plan, and outpatient clinics;

$\Delta$ Potential to expand into pharmaceutical care; and

$\Delta$ Shared values.

However, Knudsen cautioned, there are also pitfalls to be avoided. Among them: loss of focus and control, dependence on others outside pharma$\mathrm{cy}$, financial and political risk of operating such a large system, and over-commitment to certain projects.

He said Scott and White attempts to minimize these problems by defining a set of core values that everyone lives by: 1. Assembling respected leadership.

2. Hiring a talented staff and letting them do their jobs.

3. Achieving mutual respect between the pharmacy and medical staffs.

4. Focusing on teamwork, not on turf battles.

5. Developing cost improvement plans through a team approach.

6. Never sacrificing quality for cost. 